\title{
Isolated tuberculosis of the spleen: A case report and review of literature
}

\author{
H. Mishra • R. Pradeep • G. V. Rao • S. Anuradha • \\ D. Nageshwar Reddy
}

Received: 7 August 2008 / Accepted: 25 April 2012 /Published online: 15 February 2013

(C) Association of Surgeons of India 2013

\begin{abstract}
Isolated involvement of spleen in tuberculosis is a rare entity, particularly among immunocompetent hosts. Herein, we present a 28-year-old man with left abdominal discomfort for 2 years who was found to have a cystic lesion in spleen on evaluation. Eventually, he was diagnosed to have isolated splenic tuberculosis with an involvement of a single lymph node at the splenic hilum. In this rare form of tuberculosis, it seems that splenectomy in addition to standard antitubercular therapy is curative.
\end{abstract}

Keywords Isolated spleenic tuberculosis

\section{Case Report}

A 28-year-old male patient presented with history of left abdominal discomfort for the past 2 years. He gave no

\footnotetext{
H. Mishra • R. Pradeep · G. V. Rao $(\bowtie)$

Department of Surgical Gastroenterology,

Asian Institute of Gastroenterology, 6-3-661, Somajiguda,

Hyderabad 500082, India

e-mail: drraogv@satyam.net

S. Anuradha

Department of Pathology,

Asian Institute of Gastroenterology, 6-3-661,

Somajiguda,

Hyderabad 500082, India

D. N. Reddy

Department of Medical Gastroenterology,

Asian Institute of Gastroenterology, 6-3-661, Somajiguda,

Hyderabad 500082, India
}

history of vomiting, fever, alteration of bowel habits, or jaundice. He had no significant weight loss. All his routine biochemical investigations were within normal limits except for an ESR of $38 \mathrm{~mm} /$ hour. Sonographic evaluation revealed an enlarged spleen, with a cystic lesion showing dense internal echoes. Pyogenic abscess was suspected. On needle aspiration, $30 \mathrm{ml}$ of thick pus was found which grew no organism on routine bacterial culture. CECT scan showed a nonenhancing hypodense lesion in the enlarged spleen with a few calcific foci within it (Fig. 1a). Another smaller hypodense lesion, suggestive of a lymph node, was noted at the splenic hilum in the tail of pancreas (Fig. 1b). At laparotomy, he was found to have a large cystic lesion on the lower pole of spleen along with multiple whitish surrounding lesions (Fig. 2a). A small cystic lesion was found near the tail of pancreas at the splenic hilum.

Frozen section examination of whitish lesions on the peritoneum over the splenic hilum revealed granulomatous lesions suggestive of tuberculosis. Distal pancreatectomy and splenectomy were performed. The cut section of the lesion showed caseating necrosis of the spleen (Fig. 2b). Antitubercular therapy was started after confirmation of the diagnosis on routine histopathology. The patient improved well and he was asymptomatic on follow-up, 14 months after surgery.

Tuberculosis of the spleen usually occurs as a part of miliary tuberculosis in immunocompromised patients and is often associated with miliary involvement of other organs including the liver. Isolated macronodular involvement of the spleen in an immunocompetent patient is a rare entity. Only one of twenty-three cases was reported by Sharma et al [1]. They usually present as fever of unknown origin and 

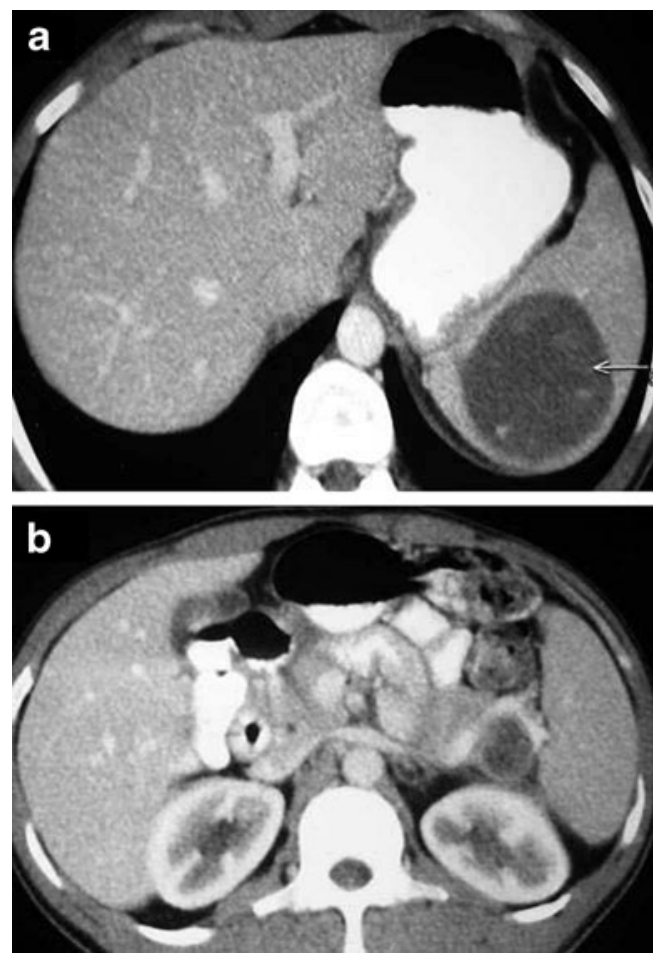

Fig. 1 (a) CECT scan showing a nonenhancing hypodense lesion in the enlarged spleen with a few calcific foci within it and (b) a smaller hypodense lesion at the splenic hilum in the tail of pancreas

sometimes as vague abdominal discomfort. Solitary splenic lesions could be either predominantly cystic or solid. Cystic lesions include posttraumatic, inflammatory and infectious
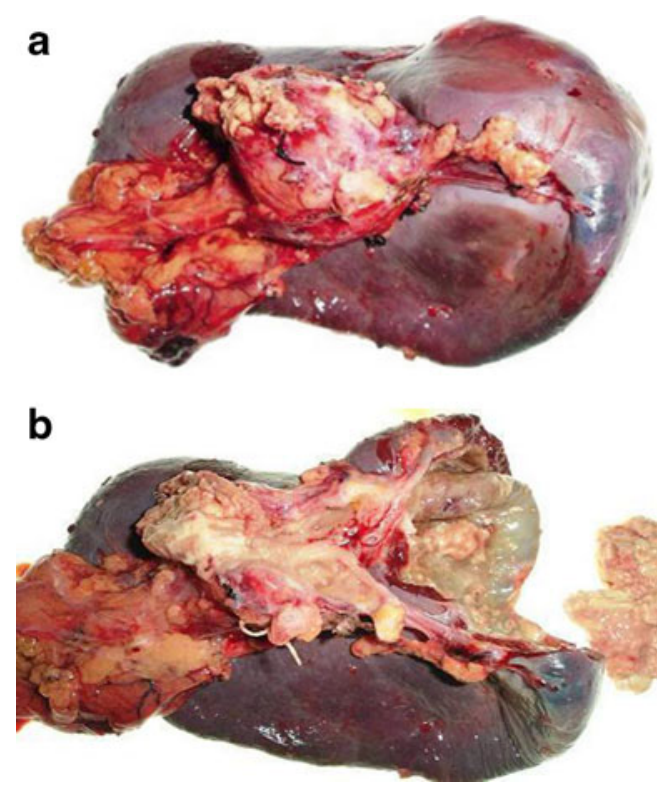

Fig. 2 (a) A large cystic lesion on the lower pole of spleen along with multiple whitish surrounding lesions and a cystic swelling at splenic hilum and (b) a cut section showing caseating necrotic material pseudocysts (e.g., tubercular, hydatid), congenital cysts, lymphangiomas, and cystic secondary metastasis [2]. Solid lesions include both nonneoplastic lesions such as hamartoma, benign tumors such as hemangioma, and malignant masses including lymphoma and angiosarcoma [2]. Mycobacterium tuberculosis (41.1\%) is the most common microorganism isolated from cases with splenic abscess [3]. Others include Mycobacterium aviumintracellulare, Streptococcus anginosus, Streptococcus milleri, Escherichia coli, and Candida albicans. Blood cultures are positive in $80 \%$ of nontuberculous splenic abscess [3]. The predisposing conditions for development of splenic abscess include AIDS, malignant neoplasm, diabetes, endocarditis, Sjögren syndrome, and previous abdominal surgery.

The radiological features of splenic tuberculosis are poorly described. Ultrasound usually reveals single or multiple focal hypoechoic lesions, representing granulomas. These lesions may be hemorrhagic or necrotic. CT scan shows isolated splenomegaly with single or multiple hypodense focal lesions with or without calcifications [4]. A focal splenic abnormality identified at sonography should be further evaluated by means of CT or MR imaging performed with and without contrast material. Due to the nonspecific clinical picture, the patient may remain undiagnosed until laparotomy. Laparoscopy has been used in a few case reports for biopsy of the spleen [5].

While a few case reports suggest treatment only with antitubercular therapy $[3,5]$, splenectomy followed by a course of antitubercular therapy [6] is considered the standard of care. In conclusion, isolated splenic tuberculosis is a rare entity. It should be considered in the differential diagnosis of patients with vague symptoms and cystic lesions in an enlarged spleen.

\section{References}

1. Sharma SK, Smith-Rohrberg D, Tahir M, Mohan A, Seith A (2007) Radiological manifestations of splenic tuberculosis: a 23-patient case series from India. Indian J Med Res 125(5):669-678

2. Warshauer DM, Hall HL (2006) Solitary splenic lesions. Semin Ultrasound CT MR 27(5):370-388

3. Arruabarrena I, Bermejo MC, Ojeda E, Cosme A, Garmendia G (1998) Incidence and clinical features of splenic abscesses, with special reference to tuberculous etiology in a general hospital. Gastroenterol Hepatol 21(10):479-482

4. Abbott RM, Levy AD, Aguilera NS et al (2004) Primary vascular neoplasms of the spleen: radiologic-pathologic correlation. Radiographics 24:1137-1163

5. Meshikhes AW, Al-Momen SA (2006) Laparoscopic diagnosis of splenic tuberculosis. Surg Laparosc Endosc Percutan Tech 16(5):355356

6. Nayyar V, Ramakrishna B, Mathew G et al (1993) Response to antituberculous chemotherapy after splenectomy. J Intern Med 233 (1):81-83 